\title{
FOREWORD
}

\section{Special Section on Ubiquitous Sensor Networks}

\begin{abstract}
Most computing paradigms of the next generation such as ubiquitous computing share a vision of capturing real world events by networking everything. Small, inexpensive wireless computing devices or sensors will be distributed at all scales throughout everyday life for sensing, inferring and understanding events. Technologies in individual fields such as sensing, transmission, networking, control and applications have been researched and developed. The fusion of these technologies is challenging but needed to make the vision come true. The term of ubiquitous sensor networks was defined to represent the fusion of the technologies for this vision by the members of two IEICE technical committees on sensor networks (SN) and on ubiquitous and real-world oriented networking (URON). These two committees were merged into a new technical committee on ubiquitous sensor networks in April, 2007, and this special section was organized by the new committee as their preliminary action.
\end{abstract}

We received 55 paper submissions and 3 letter submissions from 6 countries including the USA, Europian and Asian countries as well as Japan. All papers were peer-reviewed, and 17 papers and 2 letters were selected for publication after careful consideration and intensive discussion by the editorial committee. These papers well cover the areas of fundamental theories, component technologies, platforms and applications.

Our sincere gratitude goes to all the authors who submitted their high-quality work to this special section. Finally, the Guest Editor-in-Chief would like to thank all the reviewers and editorial committee members for their contribution to realize this excellent special section.

Members of the Editorial Committee:

Guest Editor-in-Chief: Haruhisa Ichikawa (NTT)

Guest Editors: Tomoaki Ohtsuki (Keio Univ.), Masayasu Yamaguchi (NTT)

Guest Associate Editors: Susumu Ishihara (Sizuoka Univ.), Hideyuki Uehara (Toyohashi Univ. of Tech.), Masahiro Umehira (Ibaraki Univ.), Kohtaro Ohba (AIST), Masayoshi Ohashi (KDDI), Yasutada Oohama (Tokushima Univ.), Hiraku Okada (Niigata Univ.), Hideyuki Kawashima (Keio Univ.), Narito Kurata (Kajima), Ichiro Satoh (National Institute of Informatics), Yoshito Tobe (Tokyo Denki Univ.), Kaori Fujinami (Tokyo Univ. of Agriculture and Technology), Masaki Minami (Shibaura Institute of Tech.), Hiroyuki Morikawa (Tokyo Univ.), Takaya Yamazato (Nagoya Univ.), Naoki Wakamiya (Osaka Univ.)

Haruhisa Ichikawa, Guest Editor-in-Chief

Haruhisa Ichikawa (Member) received B.S., M.S. and Dr. E. degrees in electrical engineering from the University of Tokyo in 1974, 1976 and 1989, respectively. He joined NTT laboratories in 1976, where he was engaged in fundamental research on communications software and distributed computing. He proposed and conducted many R\&D projects for software, Internet, information sharing platform, and ubiquitous networks, including business incubation. He was the executive directors of NTT Information Sharing Platform Laboratories, Network Innovation Laboratories, and the executive director of NTT Science and Core Technology Laboratory Group till August 2007. He is Professor at the University of ElectroCommunications.

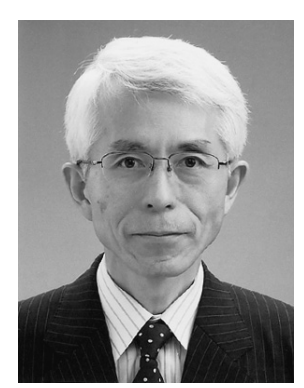

